\title{
OTIOLTOMTS
}

Revista de economía, empresa y sociedad

Dosier sobre economía colaborativa (y II)

Nuevas estrategias y dimensiones alternativas de la economía de plataforma

Coordinador: Lluís Alfons Garay Tamajón

NUEVAS PERSPECTIVAS

\section{Gambios en la oferta de Airbnb durante la pandemia de COVID-19}

\section{Gzesław Adamiak}

Nicolaus Copernicus University de Toruń, Polonia

RESUMEN Airbnb se considera la empresa de turismo de mayor valor en la historia y es el epítome de la economía de plataforma en el turismo, un sector sumido en la crisis ocasionada por la pandemia de COVID-19. El presente artículo presenta el contexto actual de la investigación internacional centrada en Airbnb, describiendo para ello el origen, el estado actual y la posible evolución de la oferta de la plataforma durante y después de la pandemia. Los datos de la oferta mundial de Airbnb en 2018, 2019 y 2020 fueron obtenidos de la plataforma por el método de extracción de datos web (scraping); estos revelaron que el crecimiento dinámico de la oferta de alojamiento se detuvo en el último año. En el transcurso de la pandemia, la oferta de la plataforma ha seguido registrando una dispersión geográfica hacia mercados menos saturados y zonas rurales. Los pisos y los apartamentos enteros siguen siendo cada vez más predominantes en la estructura de las ofertas listadas en la plataforma, mientras que el descenso en el porcentaje de ofertas de anfitriones con ofertas múltiples indica una desaceleración en el proceso de profesionalización de la oferta.

PALABRAS CLAVE Airbnb; alojamiento entre pares; economía de plataforma; economía compartida 


\title{
Changes in the global Airbnb offer during the COVID-19 pandemic
}

\begin{abstract}
Airbnb is the most valuable tourism company in history and an epitome of the platform economy in tourism. Since 2020, together with the entire tourism sector, it has experienced the crisis caused by the COVID-19 pandemic. The paper presents the context for the current international research on Airbnb by describing the origin, current state, and possible developments of the platform offer during and after the pandemic. The data on the global Airbnb offer in 2018, 2019, and 2020 comes from web-scraping the platform website. It shows that the dynamic growth of accommodation supply stopped in the last year. The platform offer has continued to disperse geographically towards less saturated markets and rural areas during the pandemic period. Entire flats and apartments have been continuously growing in dominance in the structure of the listing, while the slower growth in the percentage of multihosts' listings indicates a slowdown in the process of the professionalisation of the platform offer.
\end{abstract}

KEYWORDS Airbnb; peer-to-peer accommodation; platform economy; sharing economy

\section{Introducción}

El 10 de diciembre de 2020, Airbnb presentó su primera oferta pública de acciones en la bolsa de valores NASDAQ. No obstante el devastador efecto que ha tenido la pandemia sobre el turismo, las acciones de Airbnb se duplicaron en el primer día de su salida en la bolsa, y el valor de la empresa superó los 100.000 millones de dólares USD (Schaal, 2020a). Apenas 12 años después de constituirse, Airbnb se convertía en la empresa de turismo más valiosa de la historia y el paradigma de la economía de plataforma en el sector turístico, siendo por ello objeto de atención pública y del mundo académico. Esto no solo ocurre por el tamaño del negocio y su éxito económico, sino también por los efectos perturbadores que ha tenido en la industria de la hostelería y del mercado de la vivienda, así como por su impacto transformador en los modelos de producción y consumo turístico (Guttentag, 2019).

El presente artículo se propone presentar el contexto actual de la investigación internacional centrada en Airbnb; para ello, describimos su origen, su estado actual y la posible evolución de la oferta de la plataforma. En la primera parte presentamos un esbozo del origen y los valores fundamentales que exhibe la compañía ante su base de usuarios. También ofrecemos una descripción de la situación de la plataforma en el actual mercado de intermediarios en el alojamiento. La segunda parte presenta datos de 2018, 2019 y 2020, extraídos de la web, que reflejan el estado actual y los cambios recientemente operados en la plataforma, así como su distribución geográfica y la estructura de sus alojamientos. En la última parte, hacemos uso de esos datos para contribuir al actual debate sobre el futuro de Airbnb y de la economía de plataforma durante y después de la pandemia. 


\section{Las raíces de Airbnb y sus competidores}

Airbnb es uno de los intermediarios on-line en alojamiento más grandes del mundo. Su oferta de 5,6 millones de viviendas, apartamentos y habitaciones se extiende por casi todos los países (Airbnb, 2021). En sus campañas de marketing, la compañía se esfuerza por presentarse como una forma cualitativamente novedosa de producción y consumo de servicios turísticos. Sin embargo, los fundadores de la plataforma no aportaron una idea completamente nueva al mercado; antes bien, estos aplicaron su creatividad sobre la base de tres modelos para el desarrollo de servicios turísticos que ya existían en el mercado:

- La explotación del modelo de mercado de dos caras, en el que la plataforma actúa como la intermediaria entre proveedores y consumidores que se encuentran geográficamente dispersos. El modelo ya había sido utilizado por agencias de viajes on-line como Booking y Expedia, si bien Airbnb desarrolló una plataforma que resultaba especialmente atractiva para los anfitriones a pequeña escala.

- Basándose en el dinámico mercado del alquiler de viviendas para las vacaciones, que suele relacionarse mejor con la tendencia, muy generalizada, de hacer uso de una vivienda sin residir en ella, (Doling, 2019), Airbnb contribuyó a extender hacia las grandes ciudades el alquiler de apartamentos turísticos, que es la forma habitual de alojamiento en los centros de ocio.

- La propuesta de Airbnb se inspiró en la difusión del concepto de la economía compartida y del consumo colaborativo (Dredge y Gyimóthy, 2015). Airbnb hace posible que los anfitriones utilicen un activo infrautilizado (apartamentos o habitaciones), mientras que la plataforma recurre al relato de la idea de compartir con el fin de publicitar su servicio como una forma de actividad económica novedosa y sostenible.

No es Airbnb la única plataforma que ofrece alojamiento en casas y habitaciones privadas de manera colaborativa y digital (peer-to-peer). La Tabla 1.1 muestra una relación de las plataformas más importantes que ofrecen servicios similares. Airbnb es la plataforma de más reciente creación (si excluimos a los competidores chinos), pero es la mayor red de alojamiento colaborativo y digital. 
Tabla 1.1. Selección de plataformas de alojamiento en vivienda compartida

\begin{tabular}{|c|c|c|c|c|c|}
\hline Plataforma & Año de fundación & Sede & $\begin{array}{l}\text { Modelo de arren- } \\
\text { damiento }\end{array}$ & $\begin{array}{l}\text { Ingresos de la } \\
\text { plataforma }\end{array}$ & $\begin{array}{l}\text { Tamaño de la } \\
\text { plataforma }\end{array}$ \\
\hline HomeExchange & $\begin{array}{l}1992 \text { HomeExchange, } \\
2011 \text { GuestToGuest }\end{array}$ & París, Francia & $\begin{array}{l}\text { Puntos obtenidos } \\
\text { por los huéspedes } \\
\text { al pagar por su } \\
\text { estancia }\end{array}$ & $\begin{array}{l}\text { Cuota de socio } \\
\text { pagada por los } \\
\text { usuarios }\end{array}$ & $\begin{array}{l}450 \text { mil viviendas en } \\
187 \text { países }\end{array}$ \\
\hline Vrbo & $\begin{array}{l}1995 \text { VRBO, } \\
2005 \text { HomeAway }\end{array}$ & Austin, EE.UU. & De pago & $\begin{array}{l}\text { Suscripción paga- } \\
\text { da por los anfitrio- } \\
\text { nes o comisiones } \\
\text { por las reservas }\end{array}$ & $\begin{array}{l}>2 \text { millones de } \\
\text { ofertas }\end{array}$ \\
\hline Booking.com & 1996 & $\begin{array}{l}\text { Amsterdam, } \\
\text { Países Bajos }\end{array}$ & $\begin{array}{l}\text { Pago en hoteles } \\
\text { (principalmente), } \\
\text { viviendas y aparta- } \\
\text { mentos }\end{array}$ & $\begin{array}{l}\text { Comisiones por las } \\
\text { reservas }\end{array}$ & $\begin{array}{l}2,4 \text { millones de } \\
\text { ofertas de vivien- } \\
\text { das, apartamentos, } \\
\text { estancias en hoga- } \\
\text { res, etc. }\end{array}$ \\
\hline TripAdvisor Rentals & $\begin{array}{l}1999 \text { HolidayLettings, } \\
2004 \text { VacationHome- } \\
\text { Rentals } \\
2005 \text { Niumba } \\
2007 \text { FlipKey } \\
2009 \text { HouseTrip }\end{array}$ & $\begin{array}{l}\text { Needham, } \\
\text { EE.UU. }\end{array}$ & De pago & $\begin{array}{l}\text { Suscripción paga- } \\
\text { da por los anfitrio- } \\
\text { nes o comisiones } \\
\text { por las reservas }\end{array}$ & $\begin{array}{l}\text { > } 830 \text { mil propieda- } \\
\text { des en } 190 \text { países }\end{array}$ \\
\hline Couchsurfing & 2004 & $\begin{array}{l}\text { San Francisco, } \\
\text { EE.UU. }\end{array}$ & Gratuita & $\begin{array}{l}\text { Cuota de socios } \\
\text { en ciertos países; } \\
\text { cuota de verifica- } \\
\text { ción opcional }\end{array}$ & $\begin{array}{l}14 \text { millones de } \\
\text { socios }\end{array}$ \\
\hline Airbnb & 2008 & $\begin{array}{l}\text { San Francisco, } \\
\text { EE.UU. }\end{array}$ & De pago & $\begin{array}{l}\text { Comisiones por las } \\
\text { reservas }\end{array}$ & $\begin{array}{l}4 \text { millones de anfi- } \\
\text { triones, } 5,6 \text { millones } \\
\text { de ofertas en más } \\
\text { de } 220 \text { países y } \\
\text { regiones }\end{array}$ \\
\hline Wimdu & 2011 & $\begin{array}{l}\text { Berlin, } \\
\text { Alemania }\end{array}$ & De pago & $\begin{array}{l}\text { Comisiones por las } \\
\text { reservas }\end{array}$ & $>350$ mil ofertas \\
\hline Tujia & 2011 & Beijing, China & $\begin{array}{l}\text { De pago (también } \\
\text { en hoteles) }\end{array}$ & $\begin{array}{l}\text { Comisiones por } \\
\text { reservas }\end{array}$ & $\begin{array}{l}\text { > Un millón de ofer- } \\
\text { tas, principalmente } \\
\text { en China }\end{array}$ \\
\hline Xiaozhu & 2012 & Beijing, China & De pago & $\begin{array}{l}\text { Comisiones por las } \\
\text { reservas }\end{array}$ & $\begin{array}{l}>500 \text { mil ofertas, } \\
\text { principalmente en } \\
\text { China }\end{array}$ \\
\hline
\end{tabular}

Fuente: elaboración propia basada en Hajibaba y Dolnicar, 2018; Feng, 2019; webs de las plataformas 


\section{Oferta de Airbnb antes y después de la pandemia de COVID-19}

Airbnb no publica datos detallados datos sobre su estructura, la distribución geográfica y el uso de las ofertas de alojamiento disponibles en su plataforma. Sin embargo, desde la página web de la plataforma es posible obtener indirectamente dicha información mediante la extracción automática de datos web (web-scrapers). Los datos extraídos suelen ser empleados, por ejemplo, para investigar sobre el comportamiento de los anfitriones y los huéspedes y, asimismo, sobre cómo repercuten los alquileres de Airbnb en el mercado de la vivienda. La mayoría de los investigadores utiliza unos datasets que se compilan, archivan y publican en las webs AirDNA (comercial) e InsideAirbnb (no comercial). En la mayoría de casos, el alcance geográfico de sus análisis se limita a ciudades específicas. Los casos poco frecuentes de estudios sobre la escala espacial a nivel mundial presentan grandes variaciones geográficas en términos de dimensión, estructura e intensidad en el uso de Airbnb (Adamiak, 2019; Ke 2017).

En los siguientes apartados de este artículo, presentamos información general acerca del tamaño, la distribución y la estructura de las ofertas de Airbnb en los tres últimos años. Para ello nos basamos en tres sesiones de extracción de datos web realizadas entre setiembre y octubre de 2018; en setiembre de 2019 y en noviembre de 2020. Se utilizó el script de extracción de datos web de Slee (2018). Este no garantiza la inclusión de todas las ofertas ocurridas en un momento dado. Con el fin de filtrar ofertas inactivas, publicadas por error o duplicadas, en los conjuntos de datos (datasets) se incluyeron solo las ofertas que obtuvieron al menos un comentario. La Figura 1.1 muestra que el número de arrendamientos disponibles siguió incrementándose hasta alcanzar cerca de 4 millones de ofertas en 2019, y luego cayó un 6,5 \% hasta noviembre de 2020, por efecto de la pandemia de COVID-19.

Figura 2.1. Número de ofertas de Airbnb con comentarios en los periodos correspondientes a septiembre-octubre 2018, septiembre 2019 y noviembre 2020

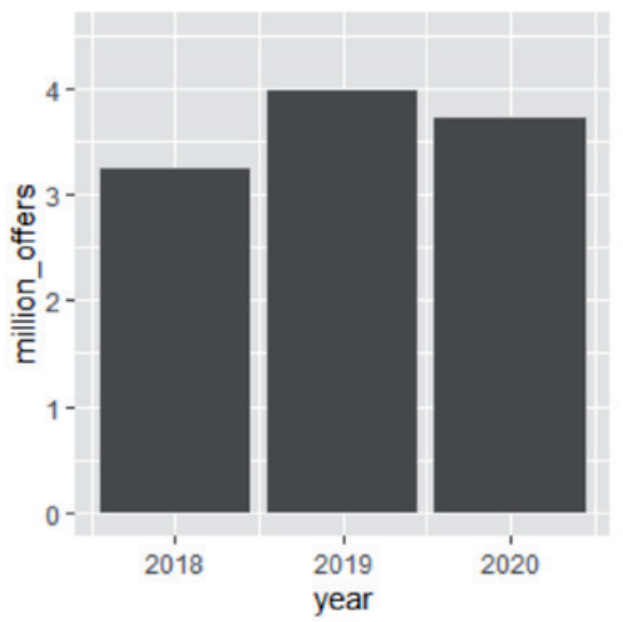

\subsection{Distribución geográfica de las ofertas}

Las mayores ofertas de Airbnb se concentran en América del norte y del sur, Europa y Asia oriental (Fig. 2.2). Una sexta parte del suministro mundial de alojamientos está localizada en los EE.UU., el país de nacimiento de la plataforma. En Europa, los mercados más grandes están en Francia, Italia, España, Reino Unido y Alemania. La lista de los diez países con mayor mercado la completan China, Brasil, México y Australia. También existen otros mercados grandes para Airbnb en algunos países pequeños de Europa, al igual que en otros países de América 
(norte y sur) y del Sudeste Asiático. El menor número de ofertas es típico de los países de África (exceptuando Sudáfrica) y de Asia central y sud-occidental. La oferta de alquileres siguió creciendo en todos los países hasta el año 2019. Se observaron tendencias distintas en cada país al registrarse caídas durante la pandemia. Al final de 2020, hubo un descenso en las ofertas de países de Europa del sur como España e Italia, llegando a niveles más bajos que en el año 2018. La mayoría de países registró un descenso en la oferta con respecto a 2019, aunque no mayor con respecto a 2018. Algunos países (por ejemplo, Brasil y Rusia) vieron un incremento continuado de su oferta de alquileres en 2020, aun con la pandemia.

Figura 2.2. Número de ofertas de Airbnb que obtuvieron comentarios en los 20 primeros países de la lista

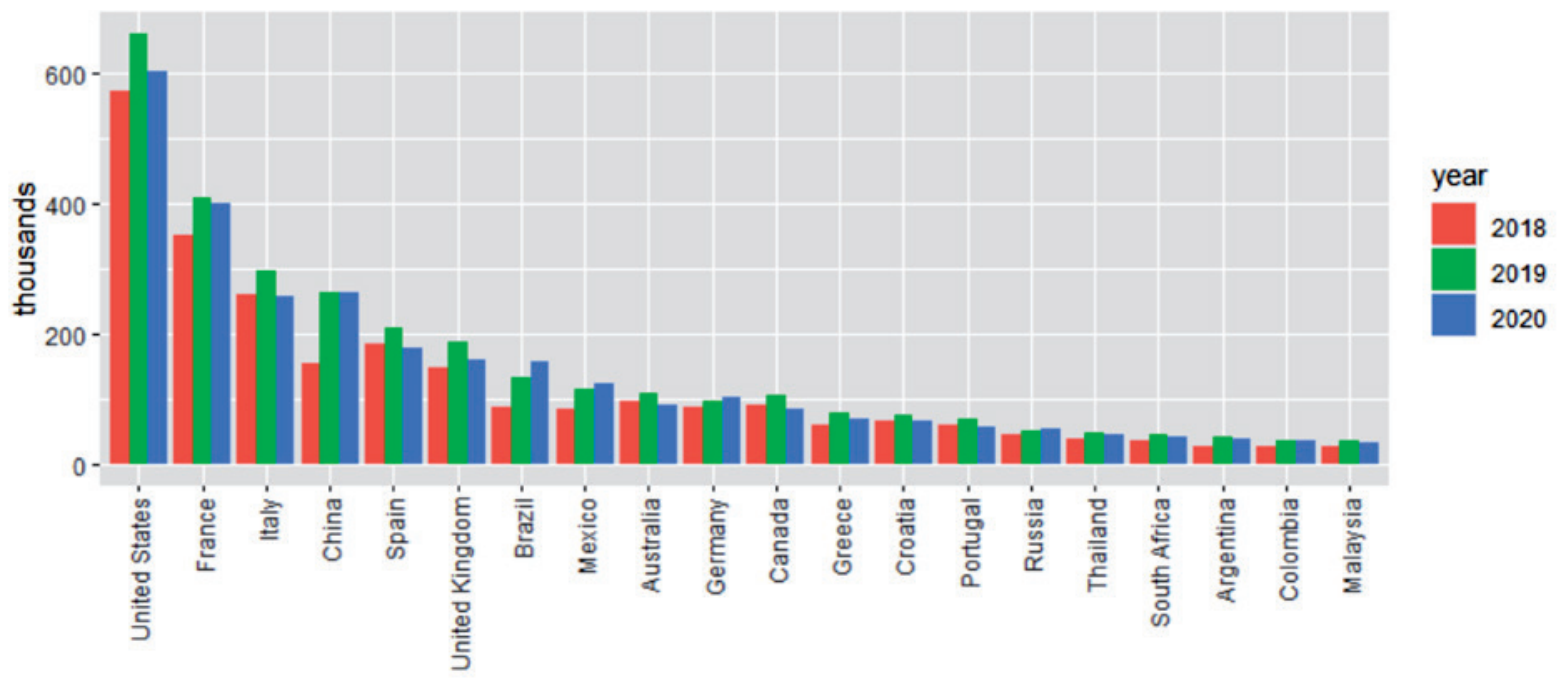

La figura 2.3 muestra el número de ofertas situadas en los mayores destinos de Airbnb. Estos lugares no son delimitados según el criterio administrativo que emplea la mayor parte de las publicaciones; en este caso, la delimitación responde a un análisis de los datos. Así, cada destino representa la concentración de ofertas de Airbnb en una zona pequeña. Los destinos fueron delimitados con DBSCAN, un método de agrupamiento por densidades (Hahsler et al., 2019) que empleó los parámetros eps=1 km y minPts=3. Los destinos de Airbnb más destacados se sitúan en las ciudades de Europa occidental (con París y Londres encabezando las ofertas), América del norte y del sur y Asia oriental. Además de las grandes ciudades, integran la lista las ciudades de Niza, Denpasar y Marbella, cuyos destinos de ocio están situados en sus respectivas periferias. 
Figura 2.3. Número de ofertas de Airbnb que obtuvieron comentarios en los 30 primeros destinos de la lista

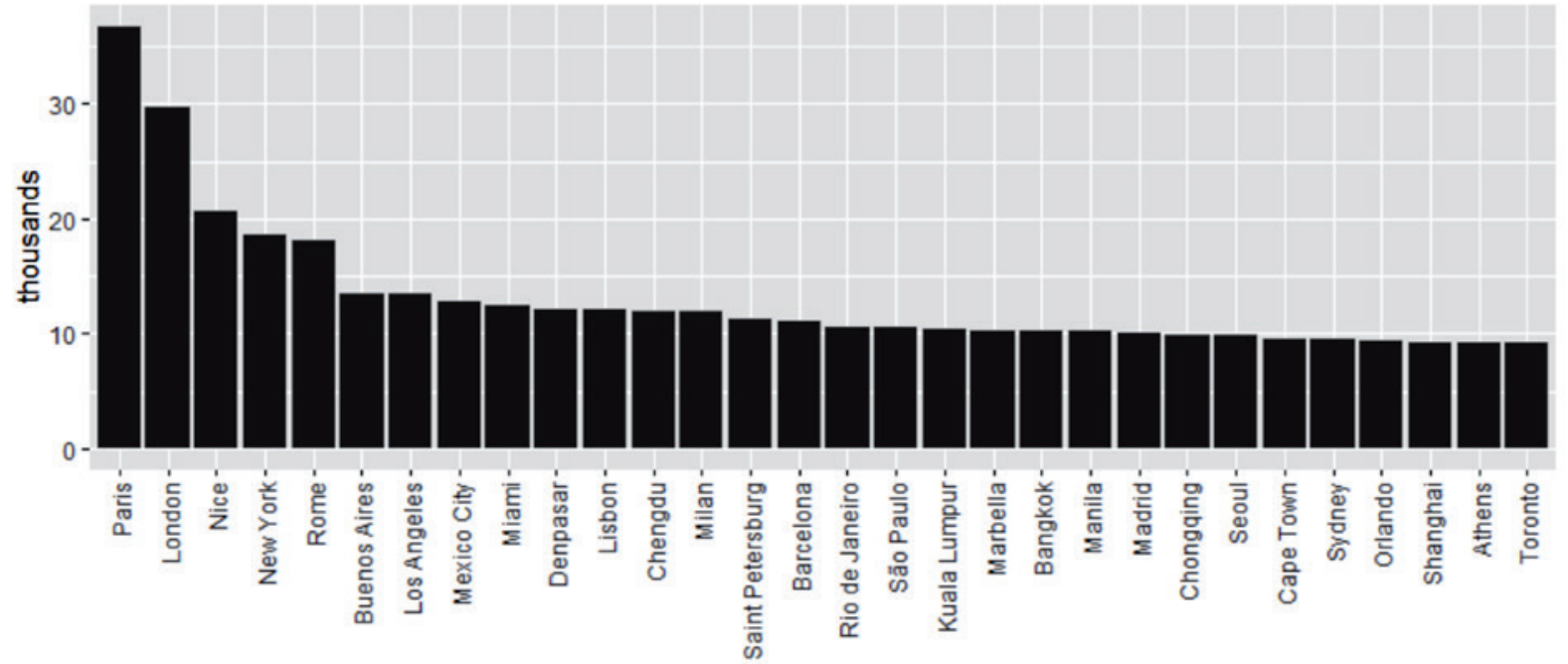

Las publicaciones suelen describir a Airbnb como un fenómeno urbano. Sin embargo, solo la mitad de las ofertas en el mundo está situada en zonas urbanizadas, siguiendo para ello la definición empleada en la base de datos geográfica de Natural Earth (2021). El resto de la oferta está situada en poblaciones pequeñas, centros de ocio y zonas rurales. En la comparativa entre países según el tamaño de sus ofertas urbanas, las ofertas de la plataforma se concentran principalmente en las ciudades de Europa oriental y de los países asiáticos. En cambio, los países de Europa meridional registran el mayor número de ofertas rurales, al ser éstas ubicadas con frecuencia en zonas costeras (Figura 2.4). En los últimos tres años, la proporción de ofertas urbanas ha descendido del 53,6\% en 2018 al 49,1\% en 2021, una tendencia que se ha vista acelerada durante la pandemia.

Figura 2.4. Porcentaje de ofertas de Airbnb situadas dentro de las zonas urbanas de los 20 primeros países de la lista

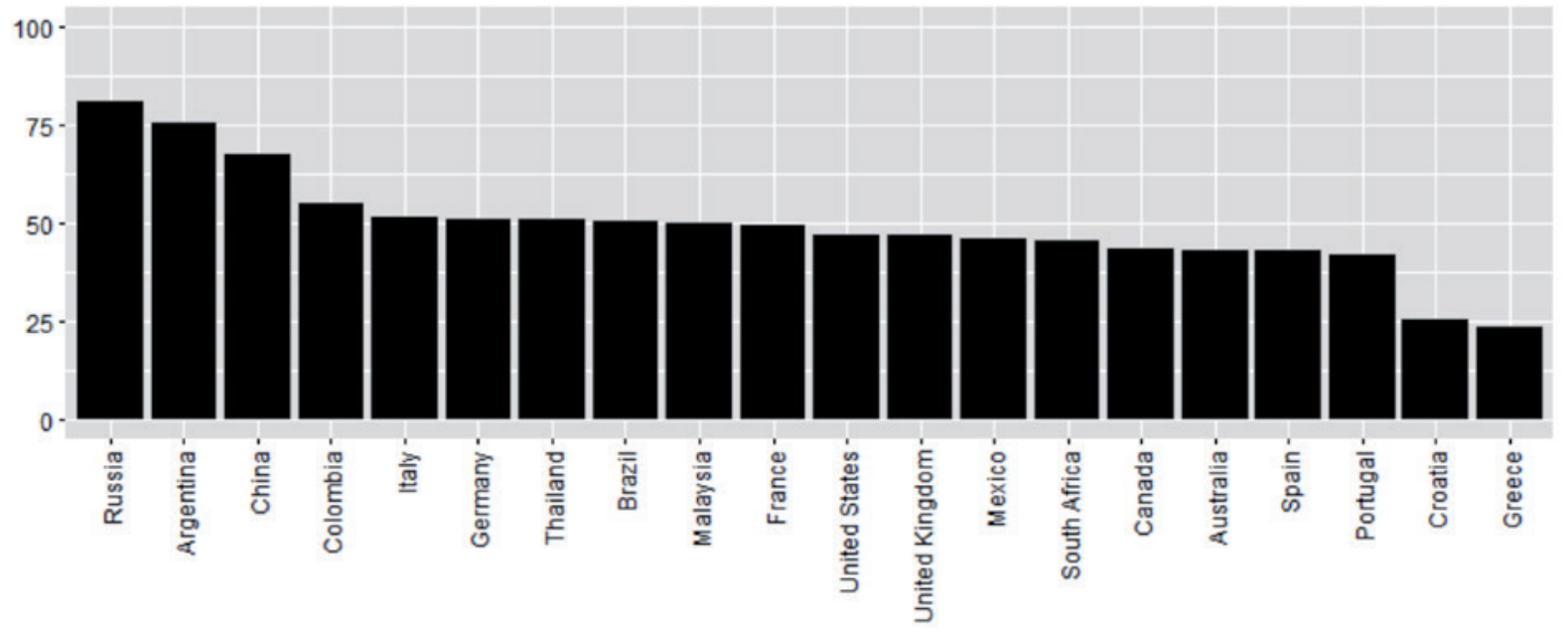




\subsection{Tipología de las ofertas de alojamiento}

Las ofertas de Airbnb tienen un uso, un valor y unas repercusiones variables según sea el tipo de oferta de alojamiento. Cuando se discute el impacto y la normativa del alquiler para estancias cortas, se suele distinguir entre anfitriones no profesionales (mamá y papá, o de par a par) y anfitriones profesionales (comerciales, con fines de lucro) (Dolnicar, 2019; Wegmann y Jiao, 2017). Los primeros arriendan una habitación en su vivienda o la casa entera cuando ellos se van de vacaciones; pero también pueden alquilar su segunda residencia. Por su parte, los apartamentos y viviendas de los anfitriones profesionales son utilizados exclusivamente para el arrendamiento, pudiendo ser ellos mismos o bien sus propietarios o bien los administradores de las propiedades de otros (Oskam, 2019). Airbnb también es utilizado como canal de distribución por los proveedores de servicios de alojamiento, los hoteles y otros lugares de pernoctación y desayuno (como el bed \& breakfast). Las normativas legales y los estudios sobre la materia distinguen entre las ofertas profesionales y las no profesionales según:

- la presencia o no del anfitrión en el apartamento

- el máximo permitido en días de alquiler anual

- el tipo de oferta (habitación o vivienda entera)

- el número de ofertas a cargo de un único anfitrión (Adamiak, 2019; Dolnicar, 2019; Oskam, 2019; Wegmann y Jiao, 2017)

En la base de datos de este estudio, se consideraron las dos últimas características arriba citadas. El tipo de oferta que predomina en Airbnb es la de viviendas o apartamentos enteros (Fig. 2.5). La proporción de este tipo de ofertas ha ido creciendo antes de la pandemia (del 72,6\% en 2018 al 74,1\% en 2019) y durante la pandemia (76,2\% en 2020). La proporción de habitaciones privadas ha descendido del 26,2\% en 2018 al 21,6\% en 2020. Las habitaciones de hotel (que son una categoría aparte desde 2019) y las habitaciones compartidas representan proporciones mínimas en el conjunto de ofertas.

Figura 2.5. Tipología de las ofertas de Airbnb que obtuvieron comentarios

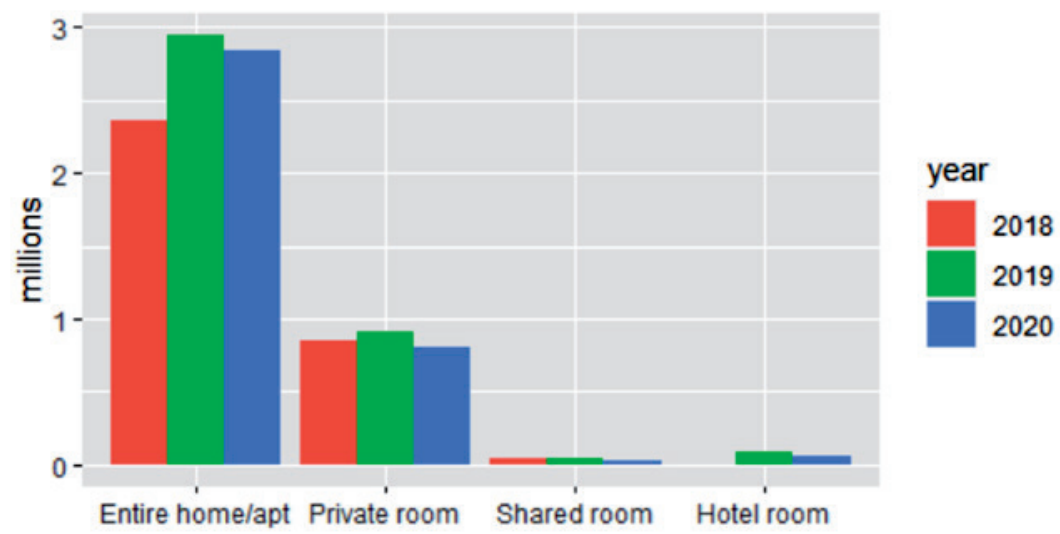

Los anfitriones con múltiples ofertas representan el 59,8\% del total de ofertas. Esta proporción representa un incremento respecto al 56,6\% registrado en 2018, aun cuando este registró una desaceleración el pasado año. El 15,1\% de las ofertas provienen de anfitriones que publicaron más de 10 ofertas, y el 5,2\% corresponde a anfitriones con más de 50 ofertas. El récord en número de ofertas publicadas por un único anfitrión es de 2.649. La Figura 2.6 muestra las diferencias entre países en relación con la cantidad de ofertas múltiples. En general, la mayor proporción de anfitriones que publican ofertas múltiples se encuentra en los mercados de Asia y Europa oriental, mientras que los anfitriones con una sola oferta son más habituales en Europa occidental y en Sudamérica. Aun así, entre los 20 primeros mercados de Airbnb, solo Francia registra una proporción mayoritaria de anfitriones con una sola oferta. 


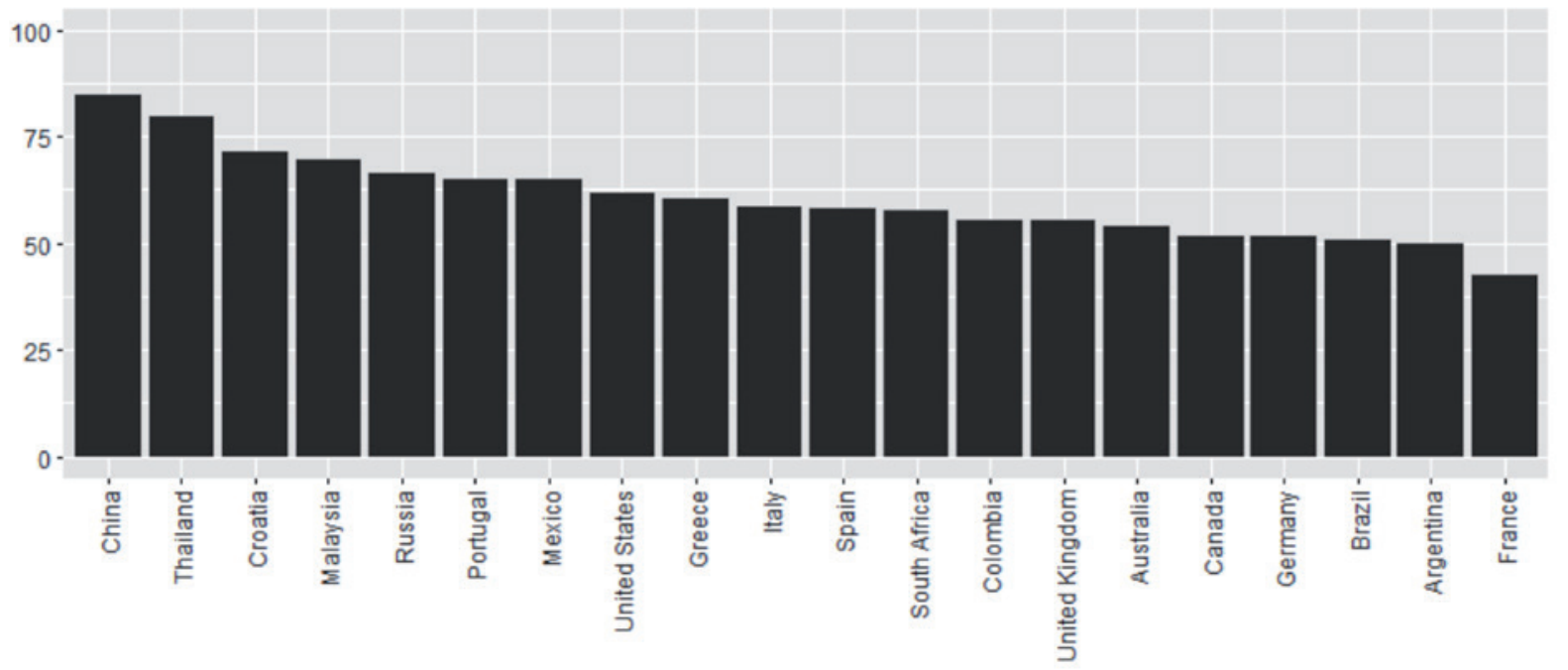

\section{Discusión sobre Airbnb y su futuro}

Antes de la pandemia, Airbnb estaba interesada en desarrollar la oferta de hoteles y anfitriones profesionales como una manera de incursionar en los viajes de lujo o de negocios por ser estos los segmentos más rentables (Dogru, Mody y Suess, 2019). La compañía adquirió otras plataformas, creó marcas para segmentos específicos (por ejemplo, Airbnb for Work y Airbnb Plus) y modificó ciertos mecanismos de la plataforma de modo que ésta fuese más conveniente para el usuario profesional. La tendencia al crecimiento rápido y desproporcionado de las ofertas de anfitriones profesionales quedó confirmada con la extracción de datos web. La estrategia de largo plazo de la compañía consistía en ampliar su oferta para los servicios turísticos más allá del alojamiento y construir una «plataforma de viajes integral», lo que fue solo en parte conseguido al añadirse productos en los apartados de «Restaurantes» $y$ «Experiencias».

La pandemia afectó a las industrias relacionadas con los viajes, de la cual Airbnb forma parte. La empresa registró una caída del 30\% en su recaudación anual y unas pérdidas netas de 459.000 millones en 2020 (Jelski, 2021) La crisis ha llevado a las plataformas a perder interés en ampliar la oferta de nuevos servicios turísticos. Por ello, la estrategia de director ejecutivo de Airbnb ha vuelto a poner el foco en los alojamientos y anfitriones microemprendedores (con múltiples ofertas), así como en las estancias de mayor duración (Schaal, 2020b). Existen estudios que pronostican futuros posibles para Airbnb tras la crisis de la industria turística. Para muchos, es posible que la oferta de alojamientos deje de crecer, principalmente por el éxito que los anfitriones profesionales están experimentando en la plataforma (Dolnicar y Zare, 2020; Zhang et al., 2020) y por el retorno parcial de la vivienda actualmente ofertada en Airbnb al mercado de la vivienda permanente (Farmaki et al., 2020; Kadi et al., 2020). Pero otros se inclinan por avizorar una renovación fortuita de la plataforma como algo que estaría ocurriendo en tiempos que requieren la distancia social: la preferencia de los clientes por alquilar pisos enteros en lugar de habitaciones de hotel (Bresciani et al., 2021).

La evidencia que ofrecen los datos revela que algunos procesos observados en la oferta de la plataforma con anterioridad a la pandemia se hicieron más visibles aún durante la crisis. Ello incluye la descentralización geográfica desde los EE.UU. y Europa sud-occidental a unos mercados nacionales menos saturados, y desde las zonas urbanas a las zonas rurales. Por lo que respecta al tipo de alojamiento, se ha registrado un mayor predominio de 
los apartamentos y viviendas en la estructura de las ofertas, mientras que los anfitriones con ofertas múltiples han dejado de crecer, lo cual confirma que la profesionalización de la oferta de la plataforma está perdiendo ritmo. Estos procesos podrían ser interpretados como una señal de que la plataforma está evolucionando más lentamente, pero también renovándose. El éxito de la salida en bolsa de la compañía parece indicar que la pandemia de COVID-19 no ha supuesto el fin del modelo de vivienda compartida sino que este seguirá redefiniendo la economía de la experiencia turística en el futuro.

\section{Referencias}

ADAMIAK, Czeslaw. "Current state and development of Airbnb accommodation offer in 167 countries". Current Issues in Tourism, 2019. DOI: https://doi.org/10.1080/13683500.2019.1696758

AIRBNB, About us, <https://press.airbnb.com/about-us/>, 2021 Disponible en: https://press.airbnb.com/about-us/ BRESCIANI, Stefano, FERRARIS, Alberto, SANTORO, Gabriele, PREMAZZI, Katia, QUAGLIA, Roberto, YAHIAOUI, Dorra y VIGLIA, Giampaolo. "The seven lives of Airbnb. The role of accommodation types". Annals of Tourism Research, 88, 103170, 2021. DOI: https://doi.org/10.1016/j.annals.2021.103170

DOGRU, Tarik and Mody, Makarand y SUESS, Courtney. "Adding evidence to the debate: Quantifying Airbnb's disruptive impact on ten key hotel markets". Tourism Management, 72, pp. 27-38, 2019. DOl: https://doi. org/10.1016/j.tourman.2018.11.008

DOLING, John. "'Not for housing' housing: Widening the scope of housing studies". Critical Housing Analysis, 6(1), pp. 22-31, 2019. DOI: http://dx.doi.org/10.13060/23362839.2019.6.1.450

DOLNICAR, Sara. "A review of research into paid online peer-to-peer accommodation". Annals of Tourism Research, 75, pp. 248-264, 2019. DOI: https://doi.org/10.1016/j.annals.2019.02.003

DOLNICAR, Sara and ZARE, Samira. "COVID19 and Airbnb - Disrupting the disruptor". Annals of Tourism Research, 83, 102961, 2020. DOI: http://dx.doi.org/10.1016/j.annals.2020.102961

DREDGE, Dianne and GYIMÓTHY, Szilvia. "The collaborative economy and tourism: Critical perspectives, questionable claims and silenced voices". Tourism Recreation Research, 40, pp. 286-302, 2015. DOI: http://dx.doi. org/10.1080/02508281.2015.1086076

FARMAKI, Anna, MIGUEL, Cristina, DROTAROVA, Maria H, ALEKSIĆ, Anna, ČASNI, ANITA Č. y EFTHYMIADOU, Fani. "Impacts of Covid-19 on peer-to-peer accommodation platforms: Host perceptions and responses". International Journal of Hospitality Management, 91(61), 102663, 2020. DOI: https://dx.doi.org/10.1016\%2Fj. ijhm.2020.102663

FENG, Linyan. "A scorecard breaking down everyone from Xiaozhu, Tujia to Airbnb". EqualOcean. < https://equalocean.com/analysis/201902091384 >, 2019

GUTTENTAG, Daniel. (2019). "Progress on Airbnb: a literature review". Journal of Hospitality and Tourism Technology, 10(4), 814-844. DOI: http://dx.doi.org/10.1108/JHTT-08-2018-0075

HAHSLER, Michael, PIEKENBROCK, Matthew and DORAN, Derek. "dbscan: Fast density-based clustering with R". Journal of Statistical Software, 91(1), pp. 1-30, 2019. DOl: http://dx.doi.org/10.18637/jss.v091.i01

HAJIBABA, Homa and DOLNICAR, Sara. "Airbnb and its competitors". In: Dolnicar S. (ed.). Peer-to-peer accommodation networks: Pushing the boundaries. Oxford: Goodfellow Publishers, 2018. DOl: http://dx.doi. org/10.23912/9781911396512-3604

JELSKI, Christina. "Airbnb says it beat its revenue projections for 2020". Travel Weekly. < https://www.travelweekly.com/Travel-News/Hotel-News/Airbnb-says-it-beat-its-revenue-projections-for-2020 >, 2021

KADI, Justin, SCHNEIDER, Antonia and SEIDL, Roman. "Short-term rentals, housing markets and COVID-19: Theoretical considerations and empirical evidence from four Austrian cities". Critical Housing Analysis, 7(2), pp. 47-57, 2020. DOI: https://dx.doi.org/10.13060/23362839.2020.7.2.514 
KE, Qing. "Sharing means renting?: An entire-marketplace analysis of Airbnb". In: Proceedings of the 2017 ACM on Web Science Conference - WebSci '17, Troy, NY, USA, June 25-28, 2017. DOI: http://dx.doi.org/10.2139/ ssrn.2902840

NATURAL EARTH. < https://www.naturalearthdata.com/ >, 2021

OSKAM, Jeroen A. The future of Airbnb and the 'sharing economy': The collaborative consumption of our cities. Bristol: Channel View, 2019.

SCHAAL, Dennis. "Airbnb Share Price More Than Doubles in First Day of Historic Public Debut". Skift. < https:// skift.com/2020/12/10/airbnb-share-price-more-than-doubles-in-first-day-of-historic-public-debut/ >, 2020a

SCHAAL, Dennis. "Airbnb CEO Brian Chesky Poised for New Era of Travel Redistribution: The Long View This Week". Skift. <https://skift.com/2020/04/24/airbnb-ceo-brian-chesky-poised-for-new-era-of-travel-redistribution-the-long-view-this-week/ >, 2020b

SLEE, Tom. Data collection for Airbnb listings. < https://github.com/tomslee/airbnb-data-collection >, 2018

WEGMANN, Jake and JIAO, Junfeng. "Taming Airbnb: Toward guiding principles for local regulation of urban vacation rentals based on empirical results from five US cities". Land Use Policy, 69, pp. 494-501, 2017.

ZHANG, Mo, GENG, Rouqi, HUANG, Yuan and REN, Shengce. "Terminator or accelerator? Lessons from the peer-to-peer accommodation hosts in China in responses to COVID-19". International Journal of Hospitality Management, 102760, 2020.

Cita recomendada: ADAMIAK, Czesław. Cambios en la oferta de Airbnb durante la pandemia de COVID-19. Oikonomics [en línea]. Mayo 2021, no. 15, pp. 1-11. ISSN: 2339-9546. DOI: https://doi. org/10.7238/o.n15.2107

Los textos publicados en esta revista están sujetos -si no se indica lo contrario- a una licencia de Reconocimiento 4.0 Internacional de Creative Commons. Puede copiarlos, distribuirlos, comunicarlos públicamente, hacer obras derivadas siempre que reconozca los créditos de las obras (autoría, nombre de la revista, institución editora) de la manera especificada por los autores o por la revista. La licencia completa se puede consultar en https://creativecommons.org/licenses/by/4.0/deed.es_ES.
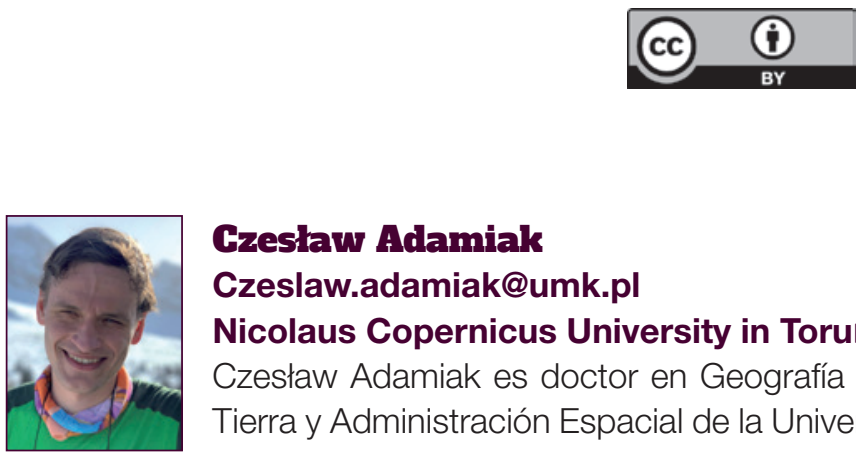

\section{Gzesław Adamiak \\ Czeslaw.adamiak@umk.pl \\ Nicolaus Copernicus University in Toruń}

Czesław Adamiak es doctor en Geografía y trabaja en la Escuela de Ciencias de la Tierra y Administración Espacial de la Universidad Nicolaus Copernicus de Toruń. Sus principales áreas de estudio incluyen la geografía del turismo, el desarrollo regional y las aplicaciones de los sistemas de información geográfica y el big data en las ciencias sociales. Actualmente sus investigaciones se centran principalmente en el papel que desempeñan las plataformas de alquiler digital P2P en el comportamiento del turismo y en la evolución de los destinos turísticos. 